\title{
PATTERNS OF DISINTEGRATION OF THE TARSUS IN THE ANAESTHETIC FOOT
}

\author{
John R. Harris and Paul W. Brand, Vellore, India \\ From the Christian Medical College Hospital, Vellore, and \\ Schieffelin Leprosy Research Sanatorium, Karigiri
}

In the absence of pain sensibility it is common for the foot to be destroyed by progressive ulceration and sepsis. The perforating ulcers of diseases such as tabes, diabetes and leprosy carry infection to the bones. Osteitis, starting at the periphery, nibbles away at the skeleton year after year until the stump of the foot becomes too small for effective weight bearing (Fig. 1). The mechanism of this type of ulceration and its prevention have been discussed in a previous communication (Bauman, Girling and Brand 1963).

In this paper we draw attention to the fact that slow progressive erosion of the foot may sometimes be interrupted by a relatively rapid and catastrophic disintegration of the proximal tarsal bones that allows the tibia to grind its way through the foot to the ground, or to become dislocated to one side and rest on the ground with the foot attached as an appendage.

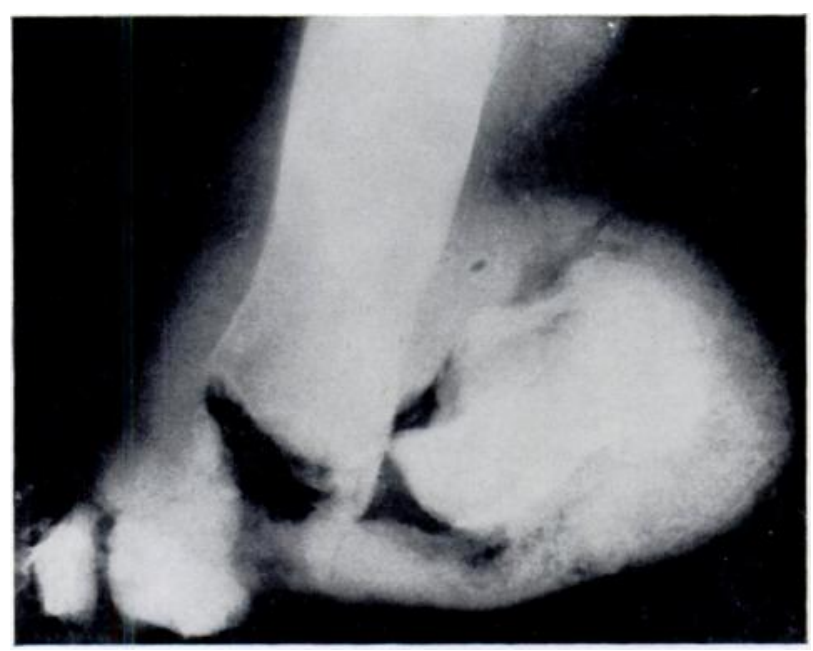

Fig. 1

The end-result of tarsal disintegration in the anaesthetic foot.

Though this proximal disintegration is not so common as peripheral erosion, its progress, once started, may be so rapid and difficult to control, and its results so disastrous, that any observation that may help in its early recognition and control becomes more important. We wish to point out that this disruption follows certain patterns that are determined largely by posture and mechanical stress in an apparently intact anaesthetic foot: these may progress to disintegration if either sepsis or further stress is added.

During a study of blood flow in the anaesthetic foot one of our research officers began to measure skin temperature in the lower limbs. His early results seemed to suggest that anaesthetic feet were, on average, warmer than normal feet when assessed after an hour's rest at $70^{\circ}$ Fahrenheit in a climatic chamber. Since these were neither recently denervated nor 
infected feet, further study seemed called for. This high average temperature proved to be a mean between some feet which were cooler than normal feet (and could thus be considered "normal" anaesthetic feet) and others which were warmer and whose warmth was of an uneven patchy distribution, often maximal on the dorsum of the foot. The localised increase of temperature was uninfluenced by antibiotics, but subsided gradually with rest in bed. On closer examination these warm areas were sometimes found to be slightly swollen. The swelling would usually subside on elevation, but sometimes left thickened tissue which on biopsy showed aseptic inflammation of the synovial membranes and joint capsules. These feet sometimes had bony ridges or thickenings on the dorsum, or were hypermobile in the midtarsal area, or tended to valgus. The radiological findings were of sufficient interest to stimulate a new study which involved the routine palpation of every anaesthetic foot in our leprosy clinics, and the taking of radiographs of all those which had patches of warmth, even though the feet appeared normal and the patient had no complaints. We have now come to rely on the patch of warmth as the carliest warning of the onset of certain patterns of disintegration and as an indication of the part of the foot at which to look for the trouble at a stage when preventive measures may still be effective.

The study reported here has made use of these and other radiographs and case histories from the Christian Medical College Hospital and the Schieffelin Leprosy Research Sanatorium. Since the patients were selected for radiography our figures have no statistical significance. We have simply analysed patterns of tarsal disintegration occurring in 147 feet and have presented illustrative cases of each type. We have also used radiographs of 310 feet showing tarsal changes that we associate with stress or sepsis, of a type that may progress to disintegration.

\section{PREVIOUS REPORTS}

Disintegration in anaesthetic feet was first described by Charcot in 1868 (Charcot 1877), who observed the rapid, bizarre and painless joint destruction which occurs in tabes. Reviews of the literature on Charcot's joints have been published by Shands (1930) and Steindler (1931) and on Charcot's joints in diabetes by Jacobs (1958) and Boehm (1962). A clear distinction between the distal erosion and the proximal destruction has been pointed out by Beidleman and Duncan (1952). We have used the term " disintegration of the tarsus " rather than "Charcot's joints" to emphasise the fact that it is often a bone injury or infection rather than joint incongruity which dominates the picture, and also to get away from Charcot's idea that this destruction may be due to "trophic" disturbances. In an account of hereditary sensory neuropathy Heller and Robb (1955) described peripheral " trophic" changes almost identical with those found in leprosy.

Most observers agree that denervated joints seem able to function normally for a lifetime unless some episode of trauma or sepsis upsets their normal congruity (Steindler 1931, Eloesser 1947). After this, repeated minor stress or even normal use of the abnormal joint causes progressive disorganisation both of the joint surfaces and of the supporting bones and ligaments. These changes are common in the lower limb because the body weight must be imposed on the damaged joints or fractured bones.

The association of vigorous activity with loss of pain sensibility seems to be the basic requirement for the development of destructive neuropathic lesions in the tarsus. In the absence of either of these two components the tarsus is preserved. It is the absence of vigorous activity which preserves the tarsus in paraplegia. The presence of pain sensibility in poliomyelitis protects the joints which are subject to unusual stresses and strains.

In leprosy, neuritis occurs selectively in the tibial nerve above the ankle and in the common peroneal nerve at the knee, with consequent anaesthesia of the foot and sparing of the deep and superficial muscles in the posterior compartment of the leg. The conditions are thus created for continued vigorous activity on an anaesthetic extremity (Shands 1930).

VOL. $48 \mathrm{~B}$, NO. 1, FEBRUARY 1966 
Sepsis-In the foot the picture of neuropathy is complicated and often dominated by the presence of sepsis. Disintegration of the metatarso-phalangeal joints is almost always the direct result of pressure ulceration which opens the joint to pathogens. Hodgson, Pugh and Young (1948) emphasised the role of sepsis in the etiopathology of the so-called neuropathic changes in bones, but they referred only to lesions in the distal half of the foot where infection almost always co-exists with neuropathy. In the tarsal area, however, the role of sepsis is not so obvious. Open wounds, if present at all, may be at some distance from the disintegrating bones and joints. Lister and Maudsley (1951), Martin (1952) and Boehm (1962) described Charcot's joints in the tarsus in the absence of infection. Whereas in the hip and the knee mechanical stress must be the main cause of disintegration, in the tarsus either sepsis or stress may play the dominant role. In some cases in which mechanical factors are dominant, recent or nearby sepsis may have prepared the way by decalcification of bones or loosening of ligaments.

\section{PATTERNS OF DISINTEGRATION}

As the foot bears weight the tibia transmits force, first to the talus, then to the calcaneus and to the anterior bones of the foot. The pattern of bony trabeculae is arranged to meet the stresses of this normal weight stream. If the posture of the foot is disturbed by external forces or motor paralysis an abnormal position results, and the new weight stream will cross the trabecular lines so that minor fractures occur more readily and ligaments may rupture. The insensitive foot will accept the stresses of full weight bearing even when it is in an abnormal and unstable position, and " the greatest pathological changes correspond to the maximum of mechanical stress in the direction of greatest weight bearing " (Steindler 1931). Steindler distinguished a pattern of destruction occurring in the axis of the leg and another in the forefoot. We have observed five patterns of destruction and have named them: 1) posterior pillar ; 2) central ; 3) anterior pillar, medial arch ;4) anterior pillar, lateral arch; and 5) cuneiform -metatarsal base (Figs. 2 to 7 ).

Pattern 1: Posterior pillar (Fig. 3)-The calcaneus is the bone that is injured most frequently when a normal person lands heavily on the foot. The resulting fracture may not be clear-cut, but the result of the internal trabecular breakdown is a flattening of the bone, and often an incongruity of the subtalar joint. In the denervated foot the calcaneus is far more vulnerable for the following reasons: 1) a patient may land more heavily on his insensitive foot;2) he will continue to walk on a cracked bone; 3 ) previous or concurrent perforating ulcers in the heel may have weakened the bone by hyperaemic decalcification or may allow infection and osteomyelitis to complicate the fracture.

The patient comes walking to the clinic. He commonly has a perforating ulcer or the scar of an ulcer under his heel. He may complain of a swollen heel or a vague pain in the area, or the alert physician may notice the deformity or feel the local warmth when the patient comes for routine medical treatment. Careful questioning may elicit a history of injury, but rarely of a fall from a height. Radiographs in early cases may show a crack in the bone or flattening without any obvious crack. This results in new weight-bearing stresses which are now transverse to the trabecular alignment. In later cases there may be gross deformation of the calcaneus and a progressive upward movement of the posterior part of the bone. Steindler (1931) published a case of this type. Once the damage has been started and the bone is flattened, there is a great tendency for progressive change of shape. This is probably due to repeated injury or to sepsis in a bone which never has a chance to recover and consolidate after one injury before the next is upon it. It behaves almost as if the bone has become soft and is bending under the thrust of the body weight and the pull of the tendo calcaneus. Once the calcaneal recurvatum has become established the tendo calcaneus loses its leverage on the forefoot, and the weight of the body is transmitted neither to the forefoot nor to the heel, but straight down through the centre of the foot. Vertical compression forces may then make short work of the already fragmented and attenuated bones.

THE JOURNAL OF BONE AND JOINT SUR GERY 


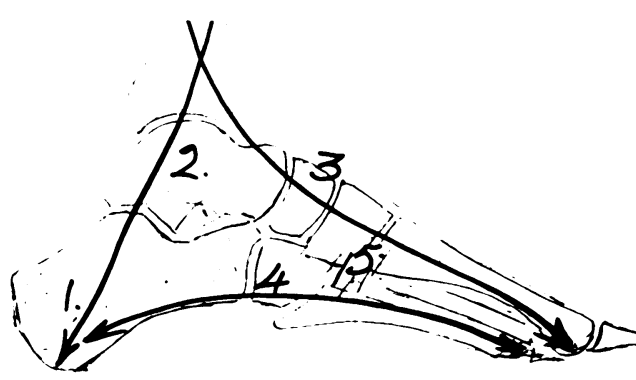

FiG. 2

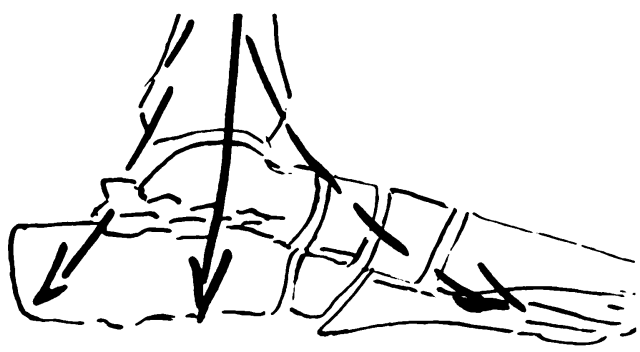

FIG. 4

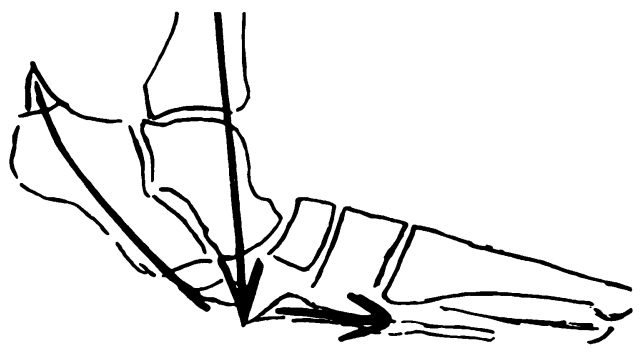

Fig. 6

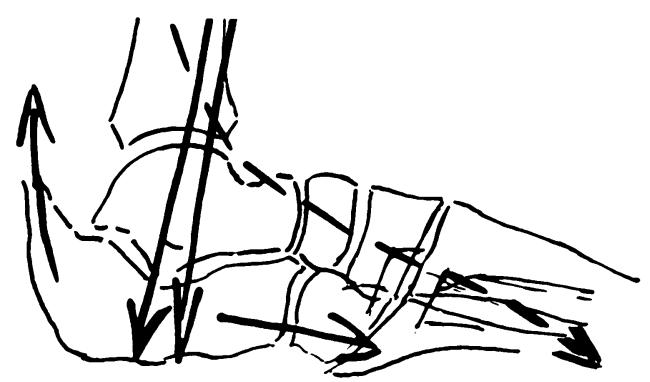

FiG. 3

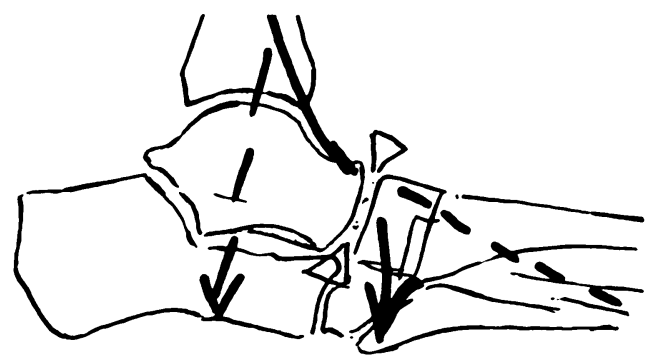

Fig. 5

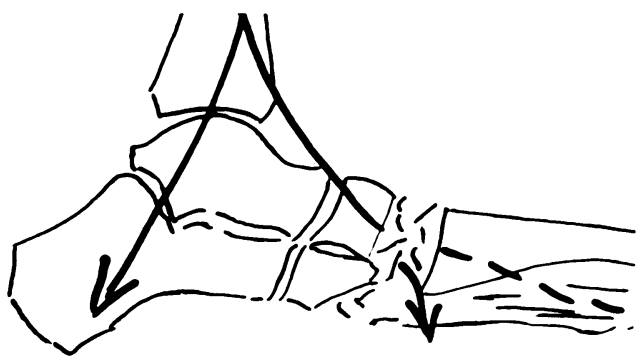

FIG. 7

The distribution of stresses in the foot and the patterns of tarsal disintegration. Figure 2-Distribution of stresses. Figure 3-Pattern 1. Collapse of the posterior pillar; effective thrust on anterior pillar now vertical. Figure 4-Pattern 2. Disintegration of the talus from subtalar incongruity. Figure 5-Pattern 3. Fracture and disintegration of the navicular bone leading to flat foot and pressure under the centre of the foot. Figure 6-Pattern 4. Destruction of the lateral ray allows plantar-flexion of the calcaneus and talus and acute dorsiflexion and subluxation of the anterior pillar. Figure 7-Pattern 5. Fracture and disintegration of the cunciform bones.

\section{CASE REPORTS}

Case 1-A carpenter of fifteen had had leprosy for three years. It had started with anaesthesia, and was bacteriologically negative. Two years before admission he had had an ulcer under the left forefoot. He was ejected from a train because of the leprosy and had to walk six miles on a hot road. A blister developed under the heel. Three months later an abscess developed and sinuses formed on the lateral side of the heel. Since then there had been chronic infection, and operations for excision of sinuses and removal of a spur had been done. Radiographs showed flattening of the tuber-joint angle and early calcaneus recurvatus (Fig. 8).

It is interesting that there was no history of injury sufficient to break the bone, and yet the bone bent. Also, the trabecular pattern of the calcaneus has altered in conformity with the new pattern of stress: the longitudinal trabeculae are faint, and the transverse ones are realigned and hypertrophied. The adjustment has of course occurred in an anaesthetic foot.

Case 2-A man of thirty-five had had leprosy for seven years, beginning with anaesthesia of the extremities. For several years there had been ulcers and sinuses on the lateral side of the right heel. The ulcers healed after treatment in plaster, but the chronic infection combined with continued walking caused fragmentation and destruction of the calcaneus (Fig. 9). When the infection had been 


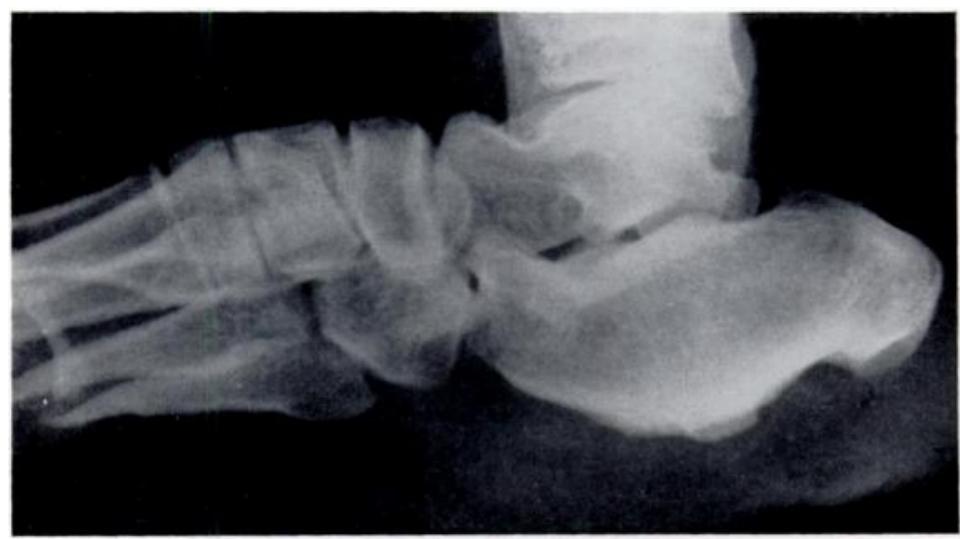

FiG. 8

Case 1-Radiograph showing early calcaneus recurvatus in a lad of 15 years, following chronic infection. Note the alteration of the trabecular pattern.

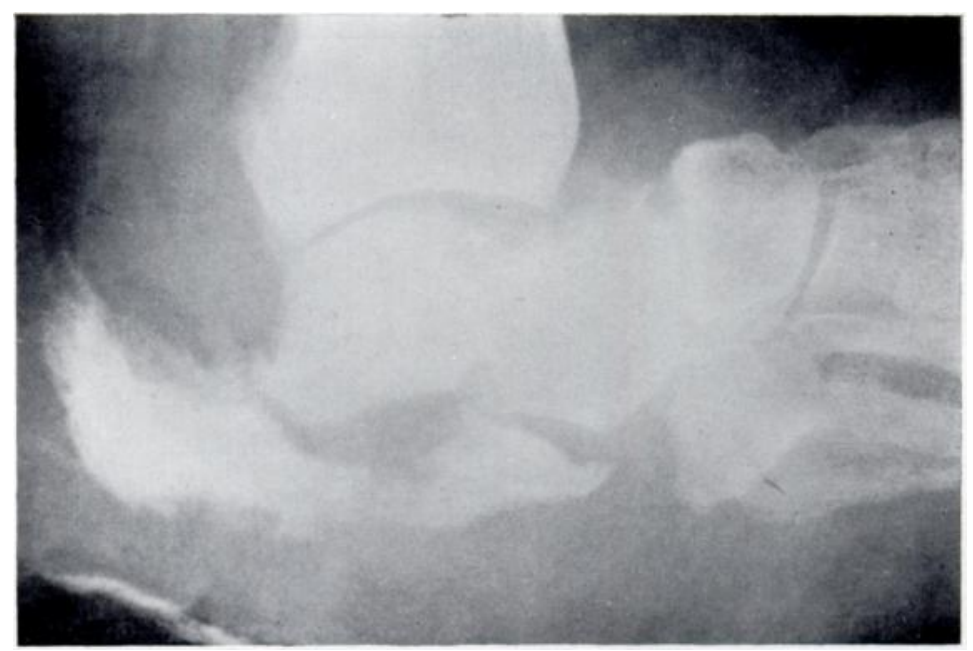

Fir. 9

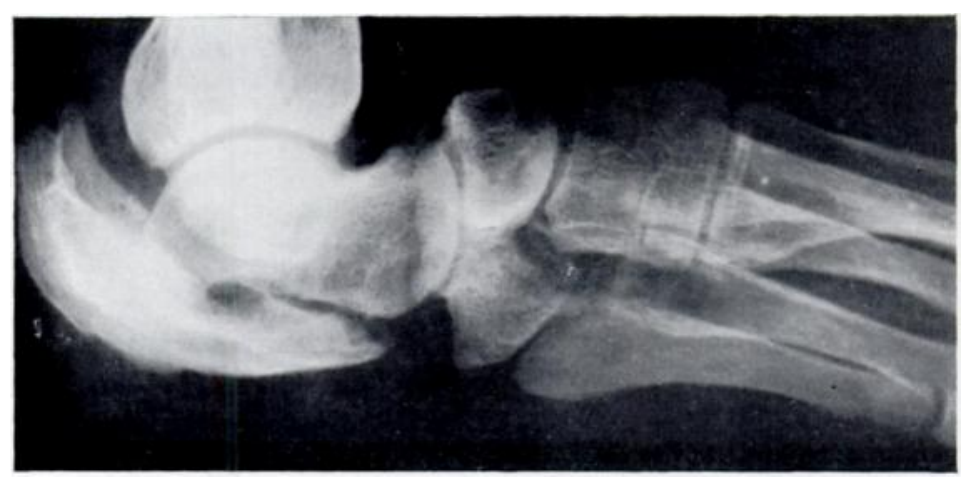

Fig. 10

Case 2-Radiographs showing progressive flattening and recurvatus deformity of the calcaneus following infection. Figure 9-At the time of active infection Figure 10-Consolidation after complete healing. Loss of calcaneo-cuboid stabilisation has allowed the forefoot to subluxate dorsally at the talonavicular joint. The final shape of the bone seems to be determined by the pull between the tendo calcaneus and the cuboid around the body of the talus.

THE JOURNAL OF BONE AND JOINT SURGERY 
completely cleared up and the foot had been protected with a special shoe the bone consolidated in the deformed shape (Fig. 10). By then, however, the forefoot had subluxated dorsally at the talonavicular joint and had become useless for weight bearing.

Pattern 2: Central (body of talus) (Fig. 4)-It is not common for the talus to be the primary focus of disintegration. Occasionally a patient may suffer a fracture of his talus and then continue to walk on it. More commonly the talus may disintegrate because of subtalar incongruity. It is interesting that the upper surface of the talus and the ankle joint are rarely damaged in leprosy. This is in contrast to tabes where ankle joint involvement is relatively common. The ankle joint is supplied partly by a nerve which arises from the tibial nerve above the point where it is usually damaged in leprosy. The nerve supply to the subtalar joint leaves the nerve in the affected segment. When we have removed the remaining fragments of a disintegrated talus we have usually found an intact shell of bone above, under the normal cartilage.

The sequence of events may be as follows: first there is flattening of the calcaneus with or without obvious fracture. Then degenerative changes start in the subtalar joint because of incongruity of the joint surfaces. This may persist for months or years before subtalar instability suddenly begins to increase. The talus itself is eroded from below and seems to melt away leaving its upper articular surface intact as part of a normal ankle joint. When the body of the talus is lost the foot becomes completely unstable and may turn over completely into valgus with the tibia resting directly on the ground.

Although the destruction of the talus seems to be due entirely to mechanical stress and incongruity it is possible that open ulcers in the forefoot may contribute to decalcification of proximal bones or loosening of ligaments by extension of forefoot sepsis along tendon sheaths and fascial planes that pass proximally in the region of the sustentaculum tali. This may also be a factor in medial arch collapse (pattern 3).

\section{CASE REPORTS}

Case 3-A youth of sixteen came in August 1961 for treatment of an ulcer under the head of the fifth metatarsal bone. The foot was treated in a plaster boot and later protective shoes were provided. The next year there was an ulcer under the heel of the same foot. A plaster was applied but after one month it was cracked in a fall. The patient walked with pain in the cracked plaster, and on removal of the plaster the ulcer was found to be healed but the ankle was swollen.

Radiographs in July 1962 showed that the bony bridge between the calcaneus and the navicular bone had probably been broken by the recent injury (Fig. 11). The lad always used to walk with his knees bent and with the foot dorsiflexed. At this stage cineradiographic studies showed only very slight movement between the talus, navicular and calcaneus, but hypermobility at the naviculo-cuneiform joint. The patient was encouraged to walk with his knees straight; the front of the ankle joint opened and there was concentration of thrust at the posterior pillar. Radiographs in February 1963 showed that the posterior part of the subtalar joint was becoming disorganised and that there was loss of depth of the body of the talus (Fig. 12). A caliper was provided, but radiographs one year later showed destruction of the body of the talus (Fig. 13). A triple arthrodesis was done: at operation thickened synovial membrane, fragments of bone and degenerating cartilage were found in the posterior part of the subtalar joint.

Case 4-A man of fifty-seven had had leprosy for twelve years and was almost cured. There was anaesthesia of the feet. He came in July 1963 with an ulcer under the great toe. At that time radiographs showed a normal talus (Fig. 14). A plaster was applied, and was kept on until September 1963. The ulcer healed well.

A few weeks after going home he twisted his foot while walking and had pain and swelling around the ankle and dorsum of the foot. He continued walking, but only for short distances. In March 1964 the patient came again with a fluctuant crepitant swelling of the foot and ankle and with extreme instability of the ankle. Aspiration gave a mixture of blood and synovial fluid. No bacteria were seen on direct smear, and no pathogens were grown on culture. Radiographs showed destruction of the talus (Fig. 15).

Arthrodesis of the tibia to the talus and calcaneus was done. At operation the body of the talus was found to be represented only by its dorsal shell and by many small fragments. There was no sign of sepsis. This case shows how a cancellous bone in an insensitive foot, once broken (even if only cracked), is pounded into fragments and absorbed.

VOL. 48 B, NO. 1, FEBRUARY 1966 


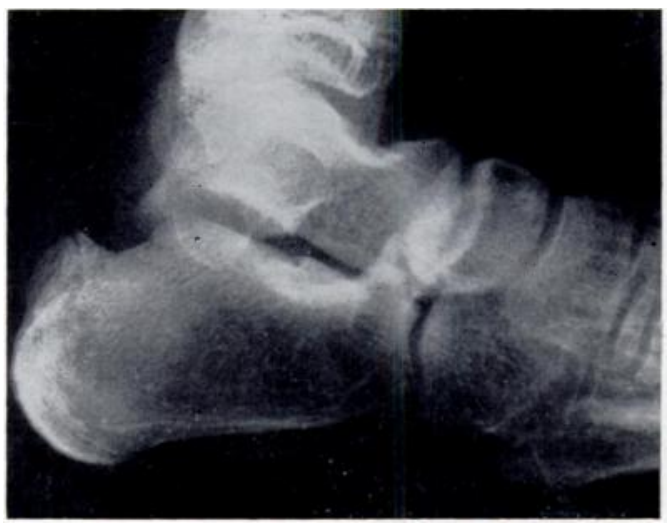

FIG. 11

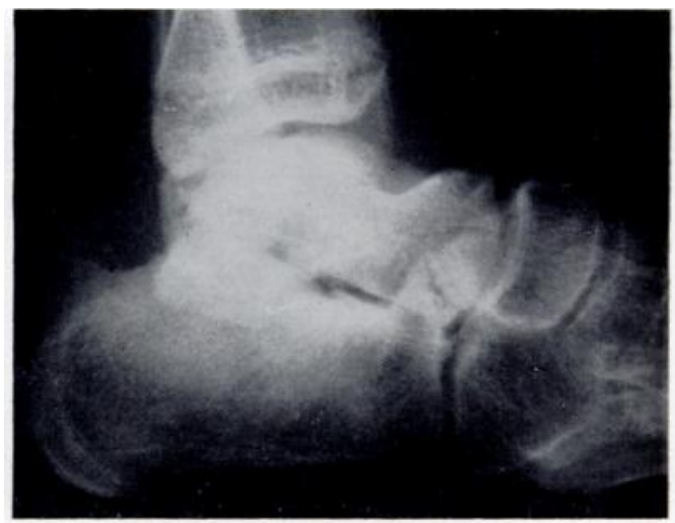

FIG. 12

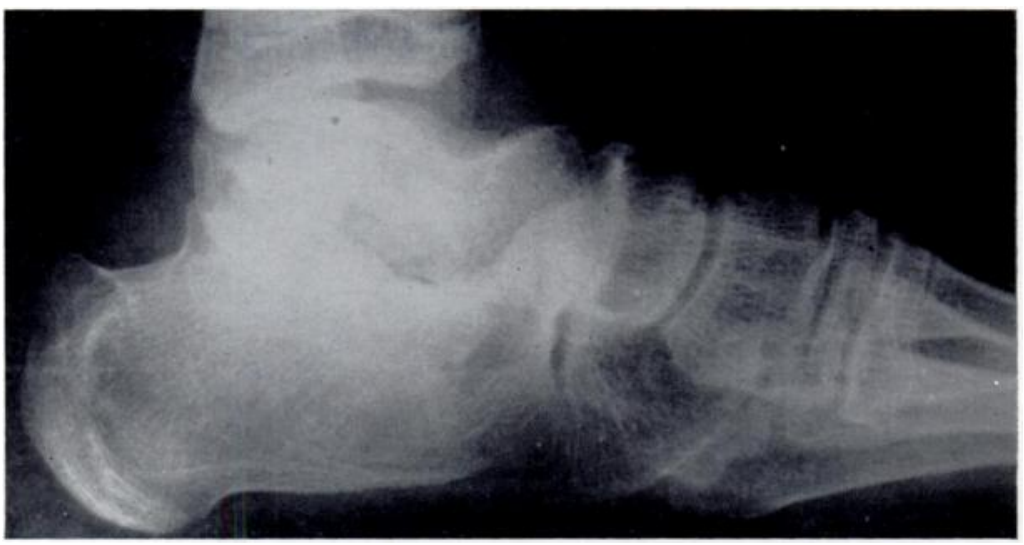

FIG. 13

Case 3-Disintegration of the talus in a youth of 16 years. Figure 11-Radiograph in July 1962 taken soon after removal of cracked plaster. There is probably a crack in the bony bridge between the calcaneus and the navicular bone. Figure 12 -Seven months later. Commencing disorganisation of the subtalar joint, with loss of depth of the body of the talus. Figure 13-One year later. Further destruction of the body of the talus with sclerosis.

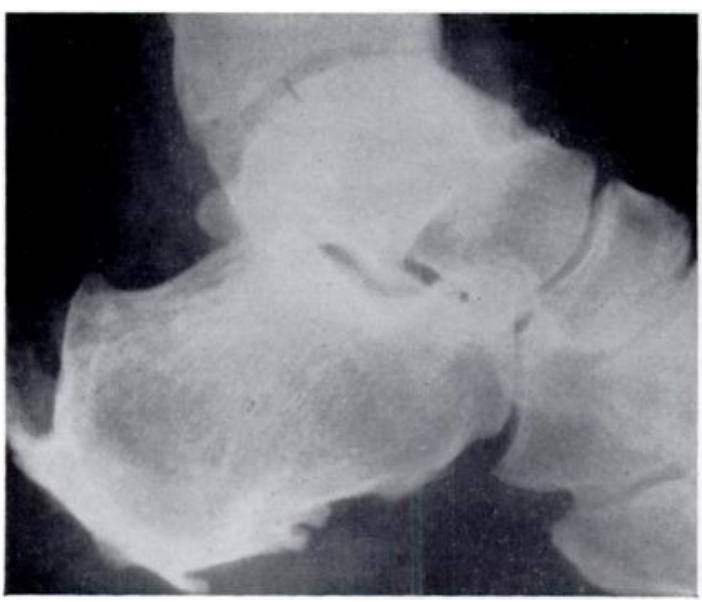

Fig. 14

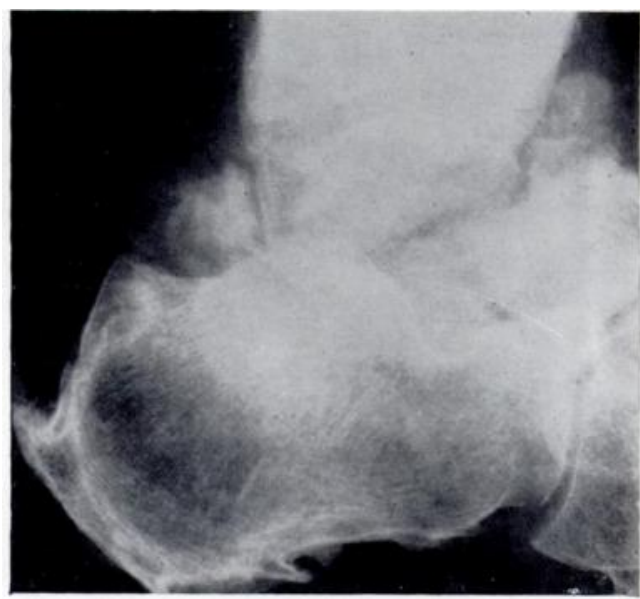

FIG. 15

Case 4-Disintegration of the talus in a man of 57 years. Figure 14-Radiograph taken in July 1963 showing normal talus at the time when the patient came for treatment of an ulcer under the great toe. Figure 15-Eight months later after treatment in plaster and minor injury after removal of the plaster. Destruction of the body of the talus. 
Pattern 3: Anterior pillar-medial arch (Fig. 5)—Collapse of the dynamic medial component of the anterior pillar was a common and consistent pattern. It corresponds to the classical forefoot collapse or " pied tabetique" seen in tabes (Steindler 1931) and diabetes (Bailey and Root 1947). It is in this pattern that the sign of localised warmth is of most value. Rundles (1945) and Martin (1952) observed localised warmth of the foot but we wish to emphasise that it is a constant and early sign and is sufficiently well marked to be picked up easily by simple palpation. Another common feature is a prominent bony ridge across the dorsum of the foot. In early cases lateral radiographs show localised sclerosis and osteophytes at the upper margins of the tarsal bones and wedging or narrowing of the upper end of the navicular and cuneiform bones. All these signs suggest strains localised to the upper surface of the tarsus.

Our engineers point out that the construction of the normal foot allows the dorsal bones to act entirely as compression members of a deep lever while the tension is taken ventrally by fascia and muscles. Any weakening or slackening of the plantar tension component, as from intrinsic paralysis or imperfect reflex coordination, would impose angulating forces on the bones and joints, localising compression entirely to the dorsal edge of the bones. The fact that all the patients with this pattern of collapse had intrinsic paralysis may be significant in this connection.

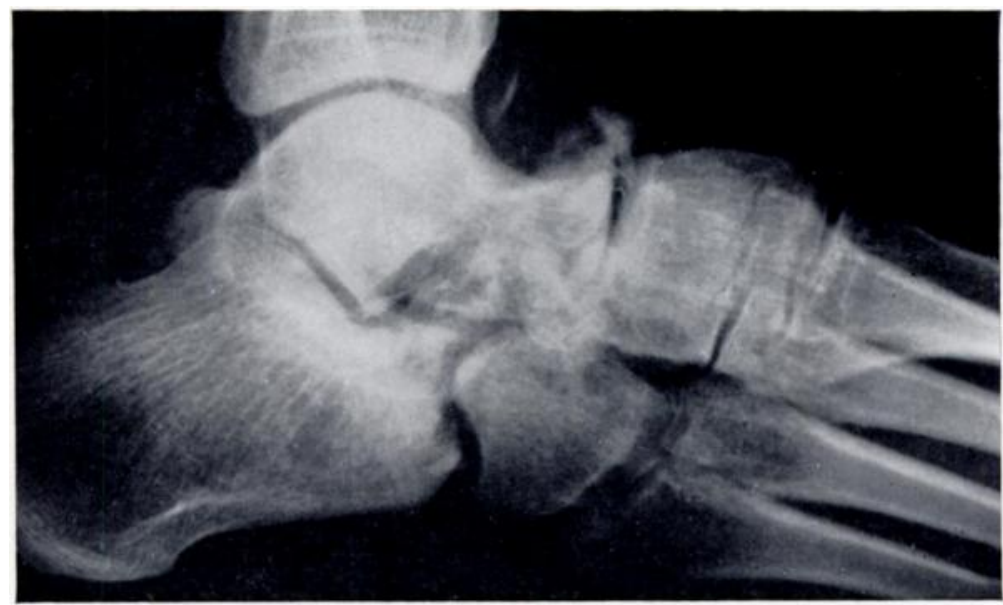

FIG. 16

Disintegration of the navicular bone. Radiograph of the left foot of a man of 23 years who had had leprosy for five years. Plantar ulcers had healed after treatment in plaster, but increasing swelling and some pain had developed after a dorsiflexion injury of the ankle. The navicular bone has disintegrated completely and the head of the talus now articulates with the cuneiform bones.

In a number of cases the condition has progressed to complete collapse of the medial arch by fracture and disintegration of the navicular bone, or occasionally of the head of the talus. In these cases a clear history is usually obtained of injury or momentary strain, followed by swelling and a sense of weakness in the foot with varying degrees of pain. This navicular fracture is seen clearly only if radiographs are taken in the very early stages.

Once the first crack has appeared, continued walking forces the head of the talus into the broken cup of the navicular. The fractured surfaces grind together and the bone is pounded until it begins to fragment and then to disintegrate completely (Fig. 16). The head of the talus may now for a time articulate with the cuneiform bones but the medial arch having lost its continuity tends to flatten and then to be reversed depriving the medial forefoot of its thrust. In this process the talus tilts forward, its head descending to form a new focus of plantar pressure and sometimes of ulceration. 


\section{CASE REPORT}

Case 5-A man of twenty-five had had leprosy for ten years. The disease was under treatment and was still mildly active. For ten years there had been partial loss of sensation in the feet. An ulcer under the heel had been recurrent over ten years. In April 1963 there was a chronic ulcer on the lateral side of the heel, and the foot was protected in a plaster for three months. A radiograph taken after

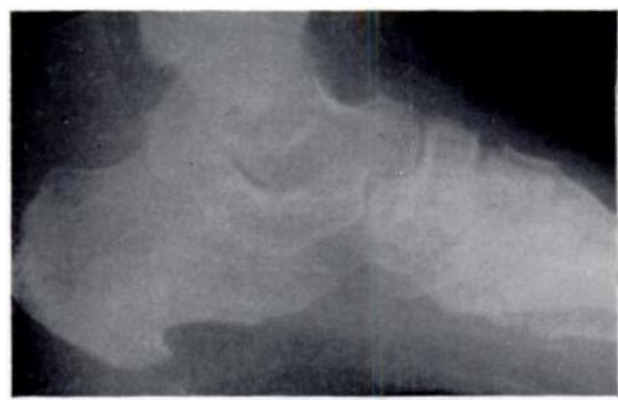

FIG. 17

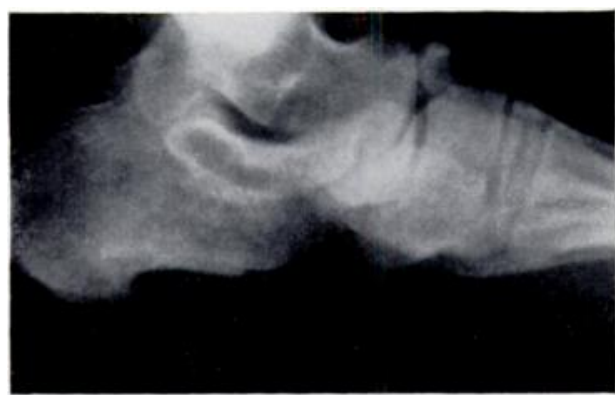

FIG. 19

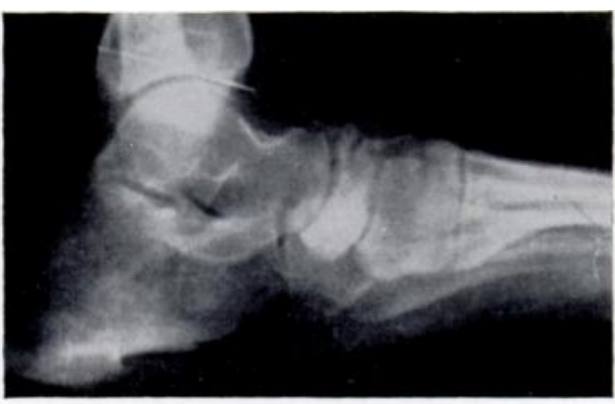

Fig. 18

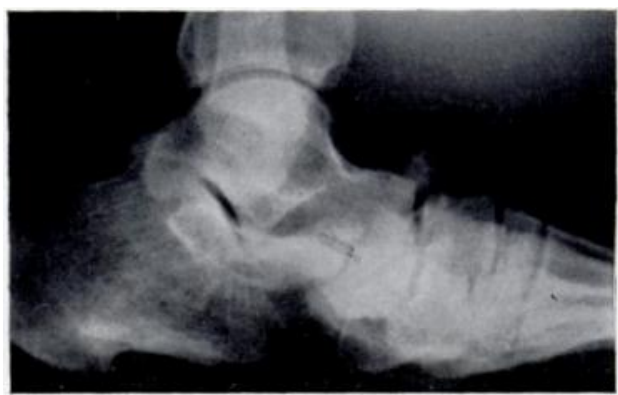

Fig. 20

Case 5-Disintegration of the navicular bone after prolonged immobilisation and subsequent walking. Figure 17-Radiograph taken in August 1963 showing early signs of stress on the superior aspect of the tarsus. Figure 18-Two months later after removal of the plaster. There is a fracture of the navicular bone. Figure 19-After two months' unprotected weight bearing. There is fragmentation of the navicular bone. Figure 20 -Four months later. Talo-cuneiform articulation developing.

this showed slight signs of stress in the upper aspect of the tarsus (Fig. 17). The foot was immobilised for another two months. Radiographs taken in October 1963 showed a fracture of the navicular bone (Fig. 18). The patient was allowed to continue walking, and two months later radiographs showed disintegration of the navicular bone (Fig. 19). Protective shoes were provided, and four months later talo-cuneiform articulation had developed (Fig. 20).

Pattern 4: Anterior pillar-lateral arch (Fig. 6)-This pattern is dominated by sepsis. A foot in the varus position has minimal mechanical strain at the midtarsus, because the medial arch is supported by the lateral arch which lies flat on the floor beneath it. In the denervated foot, however, this is a most dangerous position, because the poorly cushioned lateral bony rim of the foot is exposed to concentrated weight bearing. Perforating ulcers under the fifth metatarsal base and cuboid bone allow direct penetration of sepsis to the central bones of the lateral arch. Once the link between the calcaneus and the metatarsals, is lost, the foot becomes astonishingly hypermobile in the remaining medial tarsal joints. The pull of the tendo calcaneus may cause reversal of the arch without any further bony damage. The calcaneus tends to be drawn backwards and upwards thrusting the head of the talus downwards with acute dorsiflexion or complete subluxation of the talo-navicular joint. In other cases the sepsis may be propagated across the entire tarsus.

We have not been able to trace this pattern in the literature and conclude that this is because the characteristic deformity in both tabes and diabetes is pes planus and valgus. The 
common paralysis of the peronei in leprosy predisposes to a varus position resulting either in the above pattern of tarsal disintegration or to complete inversion of the foot with lateral ligament disruption. The patient may arrive at the clinic walking on the dorsum of his foot or even on his tibia with his foot as a medial appendage.

\section{CASE REPORT}

Case 6-A man of thirty-nine had first been affected by leprosy eighteen years previously. Three years ago an ulcer had developed on the lateral border of the left foot and had since persisted. Radiographs showed almost total loss of the lateral bones of the foot (Fig. 21).

Pattern 5: Cuneiform-metatarsal base (Fig. 7)-This is not a common pattern, but it is quite well defined. It is nearly always caused by definite violence, followed by walking on a foot with a cracked cuneiform. It has occurred from twists of the foot, and from weights falling on the dorsum. Subsequently the crack forms a weak point in the long lever of the foot which then becomes hypermobile and propagates the fracture right across the foot forming a broad flail pseudarthrosis. This type of disintegration, being primarily a fracture, responds well to simple immobilisation if it is treated in the early stages.

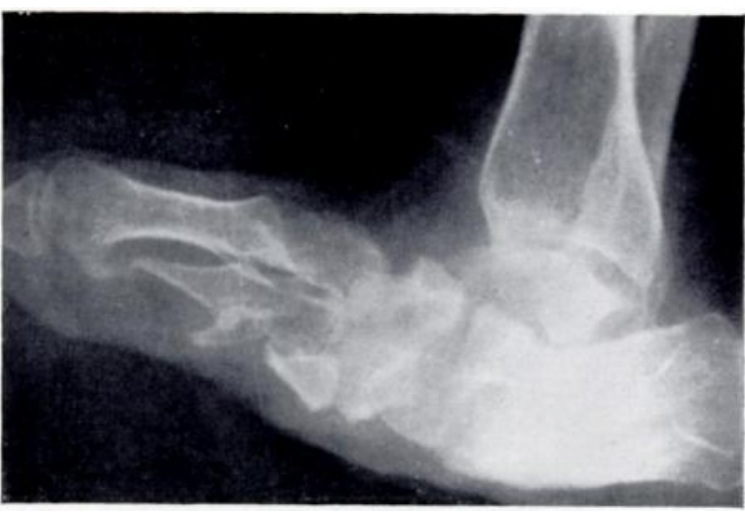

FIG. 21

Case 6-Disintegration of the lateral ray and dislocation of the medial ray. Radiographs showing almost total loss of the lateral bones of the foot. The calcaneus, released from its attachments to the cuboid bone, has subluxated backwards while the unsupported medial ray has collapsed at the talo-navicular joint. The talus is inclined almost vertically, its head resting on the floor between the calcaneus and the remnants of the lateral tarsal bones.

\section{CASE REPORT}

Case 7-A man of twenty-three had had leprosy for thirteen years and had had anaesthesia of the extremities for six. In 1960 there was an ulcer under the head of the first metatarsal bone, and next year there was a warm swelling of the right foot following a minor twisting injury. At first he walked on the affected foot, but increase of pain and swelling led to his admission. At that time radiographs showed fracture of the cuneiform bones and destruction of the cuneiform-metatarsal joints of a degree far greater than that which would be expected after a single injury (Fig. 22). The further fragmentation was probably caused by the continued weight bearing. Treatment by immobilisation in plaster was begun, and three years later there was sclerosis and healing with ankylosis (Fig. 23).

\section{THE MECHANISM OF BREAKDOWN}

There seems little doubt that these various patterns of disintegration of the tarsus in insensitive feet are examples of the same process that results in Charcot's joints in many parts of the body. The striking feature of these patterns, however, is that it is the bones which focus our attention rather than the joints. The cancellous bones of the foot, built up of a honeycomb of trabeculae all directed to resist certain anticipated forces, are all just strong enough for their job. A study of the fragile skeleton of the foot in comparison with the bulk and inertia of the human form that it must support and propel makes us wonder why tarsal disintegration is not a common occurrence even in health. That it is not so is due not only to the sound construction of the foot but to an awareness by its owner whenever the limit of its tolerance is being reached. This awareness changes with changes of tolerance. The pain threshold is lowered for example when the "fracture threshold" is lowered by trabecular weakening from disuse atrophy. We have all observed how delicately a normal patient walks for the first few days after release from prolonged immobilisation in plaster. Our leprosy 
patients suffer no such inhibitions. It is striking that in three out of nine patients whose case histories appear in this study developed trabecular collapse within a short time after release from plasters. In other cases the bones seem to have been weakened by previous hyperaemia from sepsis.

The failure of awareness of the limit of tolerance has been vividly brought home to us in our tendon transfers on anaesthetic hands. In the sensitive hand the indication that a transferred tendon has become a deforming factor is an unwillingness by the patient to use

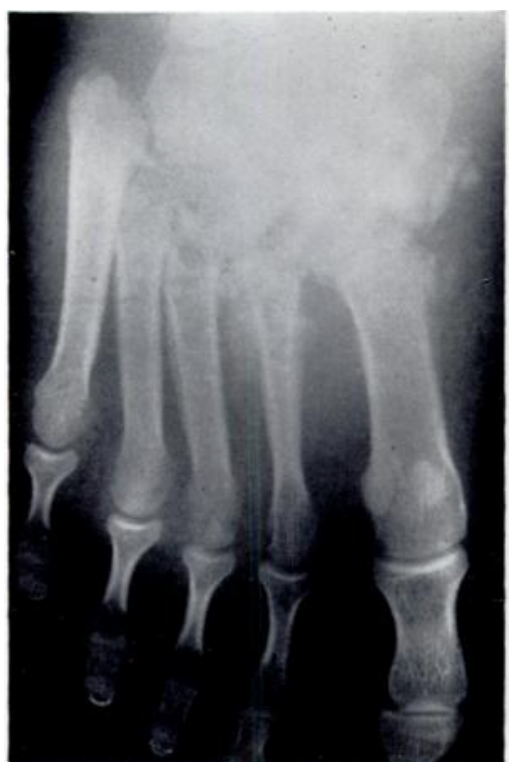

FiG. 22

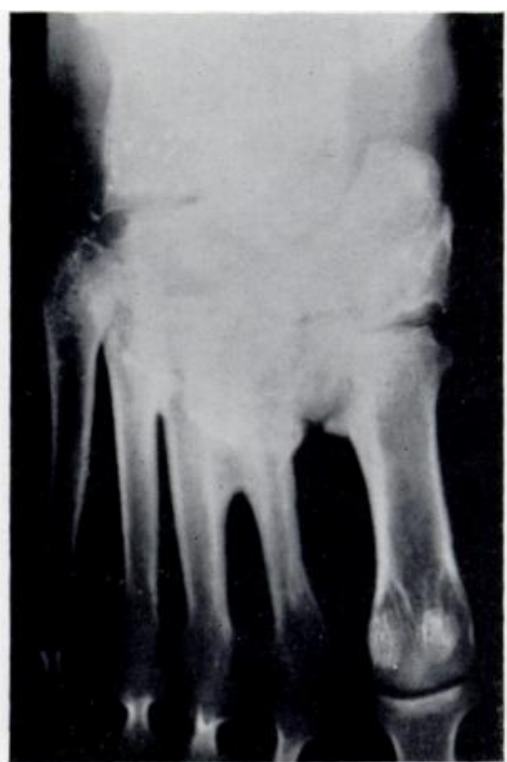

FIG. 23

Case 7-Disintegration of the cuneiform bones. Figure 22-Radiograph taken soon after admission, showing fracture of the cuneiform bones and destruction of the joint between them and the metatarsal bones. Figure 23-Three years later there is sclerosis and ankylosis.

the transferred muscle. In an anaesthetic hand the same transfer will be followed by excessive movements, subluxations and even disorganisation of joints.

In the foot it is always weight bearing which precipitates disintegration though the pattern of damage is dictated either by the habitual posture of the foot or by the location of an acute injury or infection.

\section{MANAGEMENT}

If it is not possible to restore pain sensibility to a foot, it is at least possible to limit vigorous activity. Patients with insensitive feet should understand that their feet will give them a lifetime of gentle pedestrian service, but that running, jumping or walking on rough uneven ground may cut short the useful life of the foot.

A penetrating ulcer is not to be treated lightly but as the potential destroyer of the foot. Short of complete rest in bed nothing less than immobilisation in a plaster boot is adequate.

Both the surgeon and the patient must be on the alert for the earliest signs of trouble in the tarsus. Local warmth and swelling, inversion, eversion or increasing pes planus all demand radiography, footprint studies and analysis of gait. Once it is established that there is a condition of strain in the foot, walking must be limited, and shoes adjusted until the patient can walk his limited distance without developing tarsal heat at points of stress.

We still lack experience in the management of the early stages of tarsal disintegration. The following suggestions regarding shoe modification and operative procedures should be regarded as tentative. 
Footwear. The posterior pillar-A moulded heel counter should be prescribed to spread local stress, and a microcellular laminated heel under it (like the heel of a S.A.C.H. foot) will reduce peak pressures at impact.

Medial arch-Before the arch has fallen a full arch support of firm but soft material should be used, carried up the medial side of the shoe. Once the arch has collapsed a rigid sole must be provided, pivoted on a central rocker to remove metatarsal thrust completely (Bauman, Girling and Brand 1963). A full boot is necessary to protect the foot from subtalar strains.

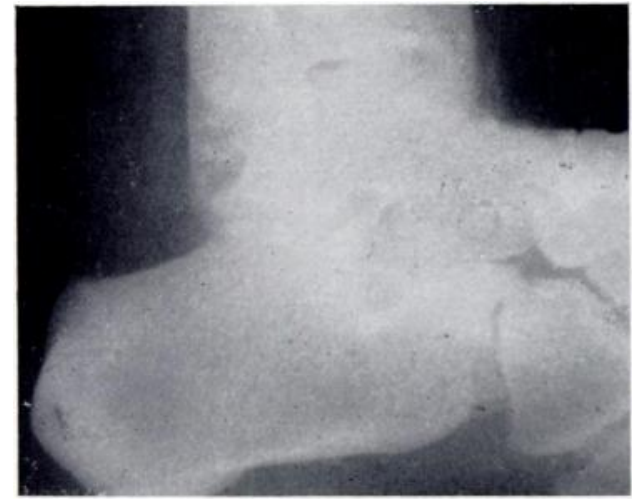

Fig. 24

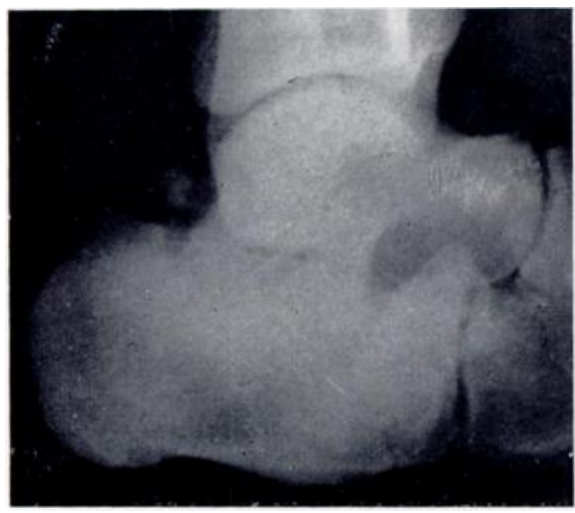

Fig. 26

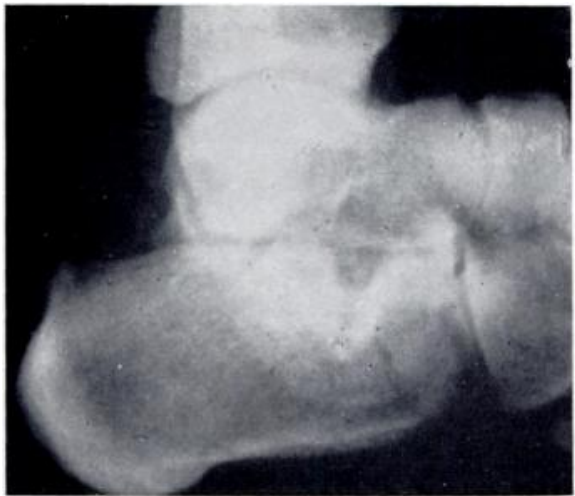

FIG. 25

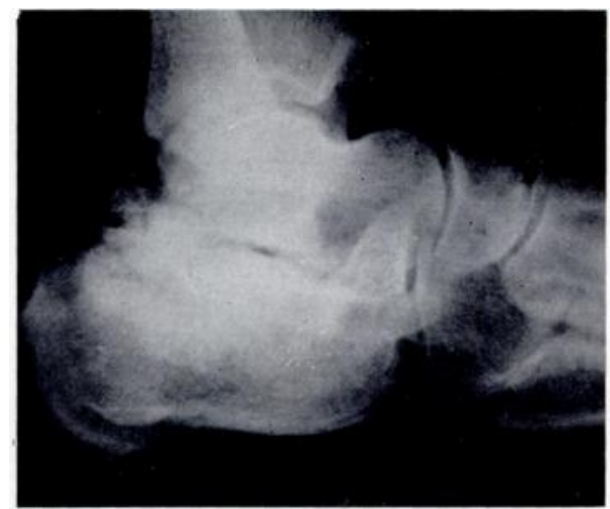

FIG. 27

Crack fracture of calcaneus healing after immobilisation in plaster. A man of 43 years had had leprosy for twenty-six years, arrested and bacteriologically negative at the time of injury. Sensory loss in hands and feet. Figure 24-Radiograph taken soon after minor injury. No fracture was then seen. Figure 25-After six weeks' immobilisation in plaster boot. Fracture of calcaneus now clearly visible; some flattening of the bone. Figure 26 -Seven months later, after further immobilisation in plaster. Healing and sclerosis. Figure 27-Three years later. No further change in the share of the calcaneus, but there are degenerative changes in the subtalar joint.

Lateral border-Surgical intervention is usually essential. Shoe adjustments are of little value in a varus foot that is in danger from lateral border stresses. Any attempt to lift the lateral border only increases the local pressure and danger of penetrating ulcers. An arch support, while relieving local pressure, may increase the deformity.

General-When definite bone damage is established, full immobilisation is imperative. Sound bony fusion will take place in a plaster but will take longer than in the sensitive foot (Figs. 24 to 27). In cases where joints are disintegrating, prolonged immobilisation may still produce arrest of the process, but failure is common and surgical fusion should not be delayed if conservative methods are not successful. Charcot's joints have a bad reputation for non-union after surgical fusion, but we have success 1) if the operation is done early, and 2) in late 
cases so long as: the joints are excised boldly back to normal-looking bone; light compression arthrodesis between Steinmann pins is used for the first month; then an unpadded carefully moulded plaster cast is used, and plaster is retained for half as long again as for similar operations in healthy feet. Even when radiographs suggest that sound union is present, we allow walking only with great caution, and palpate the foot after the first short walks. The development of local heat on walking may-sometimes draw attention to delay in union that we have missed, or may simply tell us that the patient is doing violence to his scar and needs to slow down his return to activity.

\section{SUMMARY}

1. There seem to be two distinct methods of destruction of the foot, once pain sensibility has been lost: the first is a slow erosion and shortening associated with perforating ulcers under the distal weight-bearing end of the foot. The second is a proximal disintegration of the tarsus in which mechanical forces often determine onset and progress of the condition.

2. Once the tarsus begins to disintegrate it is difficult to halt the rapid destruction of the foot. 3. It is possible to detect early stages of this condition in time to take preventive measures. Routine palpation of anaesthetic feet will reveal patches of warmth localised to bones and joints which are in a condition of strain. Radiographs of such feet and a study of posture and gait may define early changes which point to one of several possible patterns of disintegration which may follow.

4. These patterns are described and discussed and suggestions made for preventive and corrective measures.

We wish to acknowledge the help we have received at all stages of this work from a succession of colleagues and trainees in hand surgery: Dr E. Fritschi and Dr S. Gude in the studies of blood flow; Dr A. J. Selvapandian,

Dr A. Menon and Dr W. Lennox in orthopaedic surgery; Dr D. Patterson and Dr Thomas in radiology; $\mathrm{Mr}$ Howard Stalker in engineering and Mr S. Parker for his hard work at the technical level. This study has been assisted by grants from the Vocational Rehabilitation Administration, Department of Health, Education and Welfare, United States of America, and from the National Fund for Research into Poliomyelitis and other Crippling Diseases, London.

\section{REFERENCES}

Bailey, C. C., and Root, H. F. (1947): Neuropathic Foot Lesions in Diabetes Mellitus. New England Journal of Medicine, 236, 397.

Bauman, J. H., Girling, J. P., and Brand, P. W. (1963): Plantar Pressures and Trophic Ulceration. Journal of Bone and Joint Surgery, 45-B, 652.

Beidleman, B., and Duncan, G. G. (1952): Charcot Joints and Infectious-Vascular Lesions of Bones in Diabetes Mellitus. American Journal of Medicine, 12, 43.

Bоенм, H. J. (1962): Diabetic Charcot Joint. New England Journal of Medicine, 267, 185.

Charcot, J. M. (1877): Lectures on the Diseases of the Nervous System. Translated by G. Sigerson. London: The New Sydenham Society.

Eloesser, L. (1947): On the Nature of Neuropathic Affections of the Joints. Annals of Surgery, 66, 201.

Heller, I. H., and RobB, P. (1955): Hereditary Sensory Neuropathy. Neurology, 5, 15.

Hodgson, J. R., Pugh, D. G., and Young, H. H. (1948): Roentgenologic Aspect of Certain Lesions of Bone: Neuropathic or Infectious? Radiology, 50, 65.

JACOBS, J. E. (1958): Observations of Neuropathic (Charcot) Joints Occurring in Diabetes Mellitus. Journal of Bone and Joint Surgery, 40-A, 1043.

Lister, J., and Maudsley, R. H. (1951): Charcot Joints in Diabetic Neuropathy. Lancet, ii, 1110.

Martin, M. M. (1952): Charcot Joints in Diabetes Mellitus. Proceedings of the Royal Society of Medicine, 45, 503.

Rundles, R. W. (1945): Diabetic Neuropathy: General Review with Report of 125 Cases. Medicine, $24,111$. Shand, A. R. (1930): Neuropathies of the Bones and Joints. Archives of Surgery, 20, 614.

Steindler, A. (1931): The Tabetic Arthropathies. Journal of the American Medical Association, 96, 250. 